\title{
A novel approach for the preparation of nanocrystalline cellulose by using phosphotungstic acid
}

\author{
Yefei Liu ${ }^{\mathrm{a}, \mathrm{b}}$, Haisong Wang ${ }^{\mathrm{a}}$, Guang $\mathrm{Yu}^{\mathrm{a}}$, Qingxue $\mathrm{Yu}^{\mathrm{a}}$, Bin $\mathrm{Li}^{\mathrm{a}, *}$, Xindong $\mathrm{Mu}^{\mathrm{a}}$ \\ a CAS Key Laboratory of Bio-based Materials, Qingdao Institute of Bioenergy and Bioprocess Technology, Chinese Academy of Sciences, 189 Songling Road, \\ Qingdao 266101, China \\ ${ }^{\mathrm{b}}$ University of Chinese Academy of Sciences, Beijing 100049, China
}

\section{A R T I C L E I N F O}

\section{Article history:}

Received 25 January 2014

Received in revised form 8 April 2014

Accepted 11 April 2014

Available online 21 April 2014

\section{Keywords:}

Nanocrystalline cellulose (NCC)

Cellulose hydrolysis

Phosphotungstic acid $\left(\mathrm{H}_{3} \mathrm{PW}_{12} \mathrm{O}_{40}\right)$

Characterization

Thermal stability

\begin{abstract}
A B S T R A C T
In this work, a sustainable and green process to prepare nanocrystalline cellulose (NCC) from bleached hardwood pulp was demonstrated. Rod-like nanocrystalline cellulose with the size of $15-40 \mathrm{~nm}$ in width and hundreds of nanometers in length was obtained through $\mathrm{H}_{3} \mathrm{PW}_{12} \mathrm{O}_{40}$ (HPW)-catalyzed hydrolysis of bleached pulp fibers under the mild reaction conditions. Thermogravimetric analysis revealed that the resulting NCC exhibited much higher thermal stability than the partially sulfated NCC (prepared by sulfuric acid). In addition, the concentrated HPW could be easily recovered and recycled through the extraction with diethyl ether, and the recovered HPW could be reused for several rounds of cellulose hydrolysis without activity lost. These fundamental studies are of crucial importance for the development and application of NCC products/NCC-based biomaterials with good thermal stability.
\end{abstract}

(c) 2014 Elsevier Ltd. All rights reserved.

\section{Introduction}

Cellulose is one of the most abundant biodegradable polymers and it has been widely used as the reinforcing element in fiberthermoplastic composite materials at industrial scale in recent years (Eichhorn et al., 2001; Hebeish, Farag, Sharaf, \& Shaheen, 2013). Nanocrystalline cellulose (NCC) prepared from acid hydrolysis of natural cellulose, is typically a rod-like cellulose whisker which is $5-70 \mathrm{~nm}$ in diameter and hundreds of nanometers in length (Klemm et al., 2011). The size variation of NCC is highly dependent upon the origin of raw material as well as the preparation methods and conditions. Due to its nano-scale dimension, NCC has many superior properties compared to the general cellulose, such as, high aspect ratio and crystallinity, excellent optical properties and great mechanical strength (Abraham et al., 2012; Habibi, Lucia, \& Rojas, 2010; Kovacs et al., 2010; Lavoine, Desloges, Dufresne, \& Bras, 2012). Therefore, NCC has many potential applications, particularly for the production of value-added materials (Shen, Song, Qian, \& Ni, 2011). For example, nanopaper based on nanofibrillated cellulose has excellent optical transparency, high mechanical properties (tensile strength of $208 \mathrm{MPa}$ and Young's modulus of $20 \mathrm{GPa}$ ) and high gas barrier properties compared to the traditional paper and plastic substrates (Huang et al., 2013; Yousefi,

\footnotetext{
* Corresponding author. Tel.: +86 532 80662725; fax: +86 53280662724

E-mail addresses: libin@qibebt.ac.cn (B. Li), muxd@qibebt.ac.cn (X. Mu).
}

Nishino, Faezipour, Ebrahimi, \& Shakeri, 2011). Chiral nematic NCC confined within a silica host is an ideal precursor for mesoporous carbon material, which can be used as an effective electrode material for super capacitors and selective sensors (Shopsowitz, Hamad, \& MacLachlan, 2011). The nano-dimension and strength of NCC make it an ideally reinforcing material for fibrin to provide a new type of biomaterial for artificial vascular graft applications (Brown, Hu, Abu Lail, \& Zhang, 2013).

The common method to prepare NCC is hydrolysis of cellulose by mineral acids, including sulfuric acid (Beck-Candanedo, Roman, \& Gray, 2005; Fan \& Li, 2012; Tang, Yang, Zhang, \& Zhang, 2014; Wang et al., 2012), hydrochloric acid (Yu et al., 2013), phosphoric acid (Camarero Espinosa, Kuhnt, Foster, \& Weder, 2013), and their mixtures (Teixeira, de Oliveira, Mattoso, Corrêa, \& Paladin, 2010). Although this method is simple, some issues need to be addressed, such as serious equipment corrosion, large water usage and generation of a great amount of waste. Recently, many studies have been focused on the optimization of hydrolysis parameters, the avoiding of corrosion and the reduction of waste disposal. In these studies, the substitution of strong liquid acids by solid acids has been investigated for environmental and sustainable reasons. For example, previous work reported a cation exchange hydrolysis method to prepare NCC with a yield of $50 \%$, and the solid cation exchange resin can be regenerated by a post-treatment procedure (Tang, Huang, Ou, Chen, \& Chen, 2011). The major advantages of solid acid hydrolysis are the easy recovery of solid acid from the reaction mixture, the less corrosion and the relatively safe working environment 


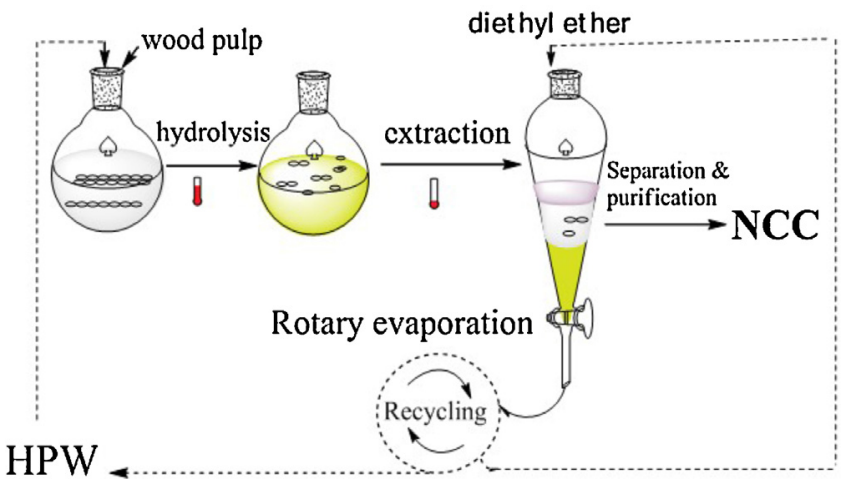

Fig. 1. The overall procedure for the preparation of NCC by using HPW (HPW: phosphotungstic acid, $\mathrm{H}_{3} \mathrm{PW}_{12} \mathrm{O}_{40}$ ).

(Huang \& Fu, 2013; Trolliet, Coudurier, \& Védrine, 2001). However, the limited contact between solid acid and cellulose significantly increases the hydrolysis time. Previous studies have demonstrated that phosphotungstic acid $\left(\mathrm{H}_{3} \mathrm{PW}_{12} \mathrm{O}_{40}\right.$, HPW) has abundant Bronsted acid sites which can break the $\beta-1,4$-glycosidic bonds in cellulose. Hence, it is an alternate catalyst of traditional mineral acids for the hydrolysis of cellulose into glucose (Drago, Dias, \& Maier, 1997; Li et al., 2012; Shimizu, Furukawa, Kobayashi, Itaya, \& Satsuma, 2009). Therefore, it is expected that NCC can be produced by HPW through controlling hydrolysis parameters, and the HPW can be easily recycled. In this work, a sustainable route to prepare NCC from hardwood pulp by HPW hydrolysis was reported, and the recovery and reuse of the concentrated HPW were demonstrated as well.

\section{Experimental}

\subsection{Materials.}

The bleached hardwood pulp was obtained from Shandong Chenming Paper Co., Ltd., China. The chemical composition of wood pulp was analyzed according to Technical Association of Pulp and Paper Industry (TAPPI) standards, and the results are shown in Table 1 (Two replicates were conducted and the average was reported). Sulfuric acid, ethanol, diethyl ether, tetrahydrofuran and phosphotungstic acid $\left(\mathrm{H}_{3} \mathrm{PW}_{12} \mathrm{O}_{40}\right.$, HPW) were purchased from Sinopham Chemical Reagent Co., Ltd. All chemicals were of analytical grade and used as received.

\subsection{Preparation of the nanocrystalline cellulose (NCC) suspension}

The overall procedure for the preparation of NCC by using HPW is given in Fig. 1. The starting wood pulp $(0.5 \mathrm{~g})$ was added to $40 \mathrm{~mL}$ $\mathrm{HPW}\left(\mathrm{H}_{3} \mathrm{PW}_{12} \mathrm{O}_{40}\right)$ solution with the desired concentration varied from $50 \%$ to $85 \%$. The mixture was heated at $90^{\circ} \mathrm{C}$ in an oil bath with mechanical stirring for the required time (15-30 h). Upon completion, the reaction was stopped by rapidly cooling the reactor in an ice bath to room temperature. Then, the resulting mixture was extracted twice with superfluous diethyl ether. As HPW could be completely extracted from water phase by diethyl ether, and HPW and diethyl ether could form a composite with large specific gravity (this composite could not be dissolved in diethyl ether or in water), three layers were formed after standing. The lowest layer (formed by diethyl ether and HPW) was collected, and the HPW was recovered after the complete evaporation of diethyl ether. After being dried at $45^{\circ} \mathrm{C}$ overnight, the recovered HPW could be reused for a second run of hydrolysis as the fresh HPW with the same procedure as described above. The upper layer was the excess diethyl ether with lower specific gravity $\left(0.7134 \mathrm{~g} / \mathrm{cm}^{3}\right.$, probably mixed with a very small amount of water). To verify the component of the upper layer, FTIR-ATR analyses of pure diethyl ether and the upper layer were carried out, and the results are shown in Fig. S1. It can be seen that the upper layer had similar FTIR spectrum pattern with pure diethyl ether except for the peaks at around 3432 and $1643 \mathrm{~cm}^{-1}$, which were associated with the mixed small amount of water. The upper layer was decanted and the diethyl ether could be recovered by simple evaporation. The middle layer mainly contained water and NCC, as well as the degraded sugars (as tested, the concentrations of glucose, xylose, and arabinose were $0.629 \mathrm{~g} / \mathrm{L}, 1.519 \mathrm{~g} / \mathrm{L}$, and $0.028 \mathrm{~g} / \mathrm{L}$, respectively. Furfural substances were undetected.) The middle layer was centrifuged $(6000 \mathrm{rpm})$ for $15 \mathrm{~min}$ at room temperature, and the supernatant was decanted. Ethanol $(40 \mathrm{~mL})$ was added to the precipitation from the middle layer, followed by $5 \mathrm{~min}$ of mixing and centrifugation $(2000 \mathrm{rpm} \times 15 \mathrm{~min})$ to remove unreacted cellulose. The suspension was washed with ethanol to remove excess acid and diethyl ether, and the NCC was collected for further centrifugation at $12,000 \mathrm{rpm}$ for $15 \mathrm{~min}$. After that, the NCC was washed by deionized water, and the washing/centrifugation cycles were repeated twice. Finally, the NCC water suspension was obtained.

For comparison, the sulfated NCC (s-NCC) was prepared by using $64 \% \mathrm{H}_{2} \mathrm{SO}_{4}$ with the solid to liquid ratio of $1: 20$ at $55^{\circ} \mathrm{C}$ for $1 \mathrm{~h}$. The hydrolysis reaction was stopped by diluting 10 -fold (volume) with deionized water. After setting, the clear top layer was decanted off and the remaining cloudy layer was centrifuged at $6000 \mathrm{rpm}$ for $15 \mathrm{~min}$ at room temperature. The resulting cellulose gel was washed with deionized water and centrifuged again at $6000 \mathrm{rpm}$ for $15 \mathrm{~min}$. The procedure of washing and centrifugation was repeated three times. Subsequently, the resulting precipitate was dialyzed with a cellulose dialysis membrane with $12-14 \mathrm{kDa}$ molecular weight cut off against deionized water until neutralization. At last, the obtained suspension was stored in a cool room for further studies.

\subsection{Dispersion studies.}

The dispersibility of the obtained NCC and s-NCC was observed by preparing NCC and s-NCC dispersions with water, ethanol, and tetrahydrofuran, respectively, at the concentration of $5 \mathrm{mg} / \mathrm{mL}$. All samples were simultaneously sonicated for $10 \mathrm{~min}$ and the dispersibility was investigated by taking pictures of the obtained suspensions immediately after sonication and after standing for $12 \mathrm{~h}$ and $48 \mathrm{~h}$, respectively.

\subsection{Drying of the NCC suspensions}

Direct water evaporation: the $2 \%$ NCC suspension was placed in an oven at $40{ }^{\circ} \mathrm{C}$ for overnight. Freeze-drying: the $0.04 \%$ and $1 \% \mathrm{NCC}$ suspensions were first frozen under $-20^{\circ} \mathrm{C}$ and then placed into a vacuum-freeze dryer for 2 days.

\subsection{Characterization.}

\subsubsection{Scanning electron microscopy (SEM)}

The wood pulp before and after hydrolysis was subjected to observation by using a scanning electron microscope (Hitachi S4800 , Japan) at $3.0 \mathrm{kV}$. Surface of the sample was coated with gold under vacuum before observation.

\subsubsection{Transmission electron microscopy (TEM)}

Dilute NCC suspensions with the concentration of $0.01 \%$ were deposited on carbon-coated TEM grids. After the samples were completely dried, the images were taken using a field emission $\mathrm{H}-7600$ electron microscope (Japan) at $100 \mathrm{kV}$. 
Table 1

Chemical composition of the original pulp.

\begin{tabular}{|c|c|c|c|c|c|c|}
\hline Material & Cellulose (\%) & Hemicellulose (\%) & Lignin $(\%)$ & Extractive (\%) & Ash (\%) & Moisture content (\%) \\
\hline Wood pulp & 87.31 & 11.26 & 1.50 & 0.00 & 0.15 & 4.4 \\
\hline
\end{tabular}

\subsubsection{Fourier transform infrared (FTIR) spectroscopy}

FTIR spectra were recorded on a Thermo Nicolet FTIR spectrometer (Nicolet 6700, USA) in the range of $400-4000 \mathrm{~cm}^{-1}$ with a resolution of $4 \mathrm{~cm}^{-1}$, and 20 scans for each sample were conducted.

\subsubsection{Thermal stability analysis}

The thermal decomposition behavior of wood pulp and NCC was studied by a thermo-gravimetric analyzer (TGA, RubothermDYNTHERM-HP, Rubotherm Co., Germany) with temperature range from room temperature to $600^{\circ} \mathrm{C}$ at a heating rate of $10^{\circ} \mathrm{C} / \mathrm{min}$ under nitrogen $(25 \mathrm{~mL} / \mathrm{min})$.

\subsubsection{X-ray diffraction (XRD) analysis}

$X$-ray diffraction patterns of the samples were characterized on a Bruker D8 ADVANCE X-ray diffractometer (XRD, Bruker Co., Germany) equipped with Ni-filtered $\mathrm{Cu} K \alpha$ radiation generated at $40 \mathrm{kV}$ and $40 \mathrm{~mA}$. The scattering angle range was $5-80^{\circ}$ with a scan rate of $4 \% \mathrm{~min}$. The crystallinity index $(\mathrm{CrI})$ was calculated according to the empirical method developed by Segal, Creely, Martin, and Conrad (1959) using the following equation:

$\mathrm{CrI}=\frac{I_{002}-I_{\mathrm{amorph}}}{I_{002}}$

where $I_{002}$ is the maximum intensity of the $\left(\begin{array}{ll}0 & 02\end{array}\right)$ lattice diffraction and $I_{\text {amorph }}$ is the intensity diffraction at $2 \theta=18^{\circ}$.

Crystal sizes were estimated using the following equation:

$D_{h k I}=\frac{0.9 \lambda}{\beta_{1 / 2} \cos \theta}$
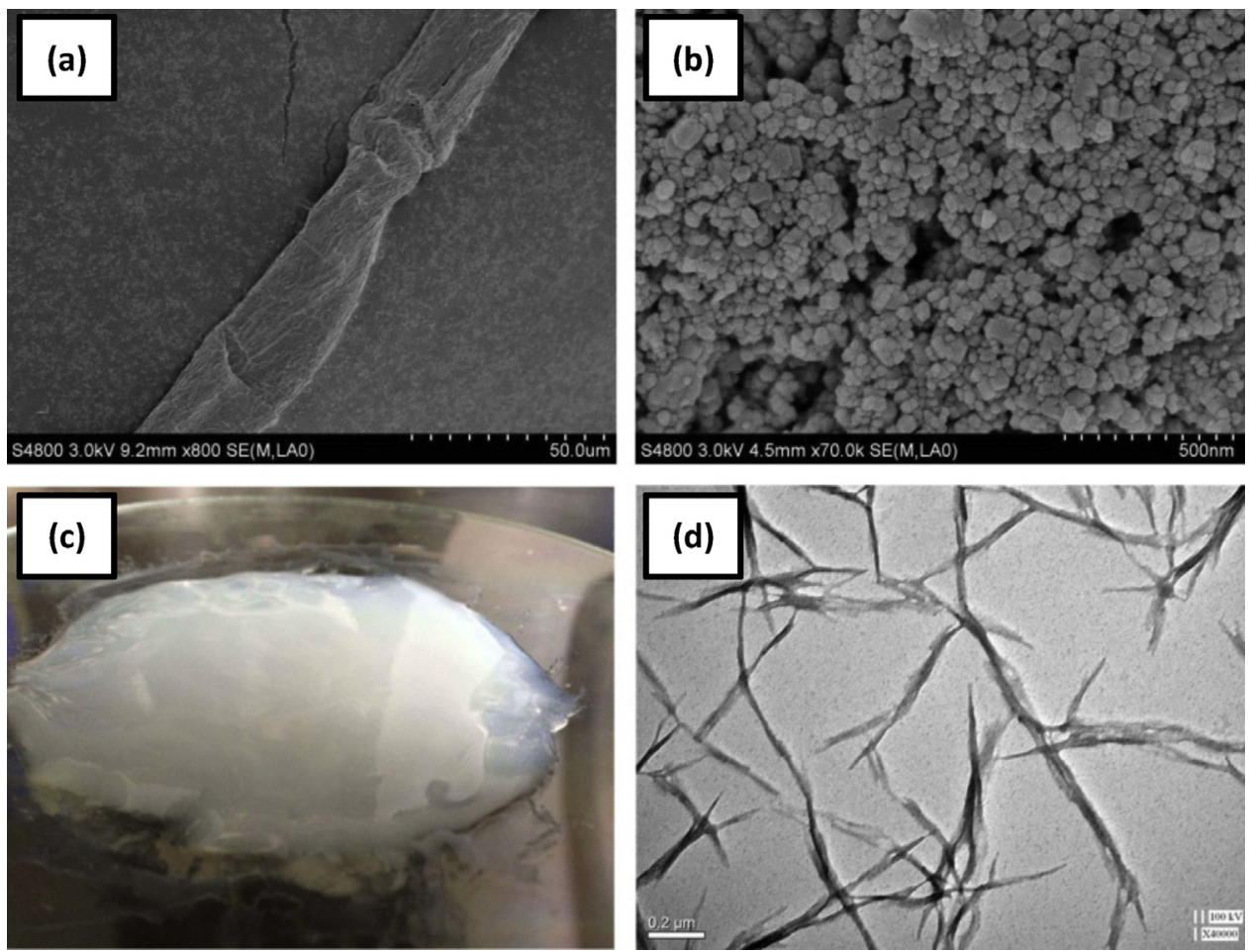

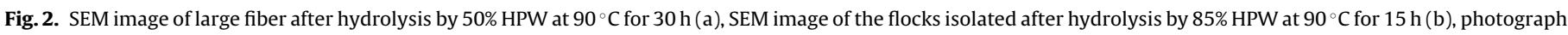
of NCC gel (c) and TEM image (d) of isolated NCC after hydrolysis by $75 \% \mathrm{HPW}$ at $90^{\circ} \mathrm{C}$ for $30 \mathrm{~h}$.

where $D_{h k l}$ is the crystal dimension in the direction normal to the diffracting planes with Miller indices of $h k l, \lambda$ is the wavelength of $\mathrm{X}$-ray radiation ( $1.54 \AA$ ), and $\beta_{1 / 2}$ is the full width at half-maximum of the diffraction peaks.

\subsubsection{Elemental analyses}

Elemental analyses were carried out in an Elemental Analyzer (Vario EL cube, ELEMNTAR, Germany) equipped with CHNS/O columns. For the CHNS column, He was used as the carrier gas, and $\mathrm{O}_{2}$ was employed for the oxidation of the samples. Before analyses, about $1 \mathrm{~g}$ samples were put in a vacuum drier at $30^{\circ} \mathrm{C}$ for $1 \mathrm{~h}$, and then the dried samples were put in a desiccator to be cooled at room temperature. The elemental analyzer was calibrated before each usage using at least five samples of the same 4-aminobenzoic acid standard.

\section{Results and discussion}

\subsection{Preparation of NCC suspension}

The hydrolysis of cellulose was attempted at different temperatures, and no cellulose nanocrystal was obtained below $90^{\circ} \mathrm{C}$. Thus, to operate the process at atmosphere pressure and to avoid excessive hydrolysis of cellulose to glucose or amorphous flocks, the hydrolysis temperature was fixed at $90^{\circ} \mathrm{C}$ in this study. Different acid concentrations and reaction time were employed to obtain NCC with high yield and the uniform dimensional appearance. It was found that at lower HPW concentration of $50 \%(\mathrm{w} / \mathrm{w})$, the reaction mixture remained pulpy even at a long reaction time for 


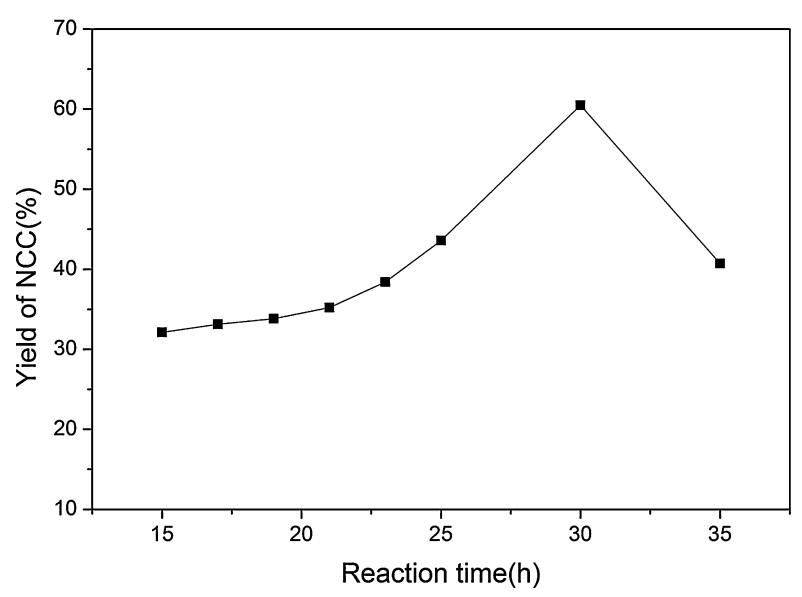

Fig. 3. Effect of reaction time on NCC yield under the same acid concentration of $75 \%$ HPW solution and reaction temperature of $90^{\circ} \mathrm{C}$.

2 days (Fig. 2a). When increasing the acid concentration to 65\% $(\mathrm{w} / \mathrm{w})$, the pulpy mixture was converted to fibers with relatively low NCC yield (20\%) after $15 \mathrm{~h}$ hydrolysis, and the yield was barely raised by extending the reaction time to $35 \mathrm{~h}$. However, when the concentration of HPW was over $80 \%(w / w)$, a tendency to form flocks was observed. This may be due to the fact that the high HPW concentration can swell cellulose crystalline so that the cellulose macromolecules were fully depolymerized and turned into amorphous nanoparticles in water (Fig. 2b), instead of forming rod-like crystals. The desired suspension of NCC gel with relatively high yield $(60 \%)$ was obtained at $90^{\circ} \mathrm{C}$ for $30 \mathrm{~h}$ by the use of $75 \%(\mathrm{w} / \mathrm{w})$ HPW, and the dimensions of the well-dispersed rod-like NCCs crystals were $15-25 \mathrm{~nm}$ in width and $600-800 \mathrm{~nm}$ in length (Fig. 2d). It can be seen from Fig. 3, the elongation of reaction time could increase the NCC yield, and the NCC yield reached 60.5\% after HPW hydrolysis for $30 \mathrm{~h}$. This yield is comparable with the one $(63.8 \%)$ obtained from cotton pulp fibers by sulphuric acid hydrolysis under the optimized conditions (Fan \& Li, 2012), while it is lower than that (76-80\%) achieved by phosphoric hydrolysis for filter paper (Whatman No. 1) (Camarero Espinosa et al., 2013). However, further prolonging the reaction time of HPW hydrolysis resulted in a decreased yield of NCC. Due to the limited contact between solid acid and cellulose, the HPW hydrolysis efficiency is much lower, and the hydrolysis time is significantly increased compared to the common acid hydrolysis (Yu et al., 2013). This problem may be addressed by introducing some suitable methods, such as ultrasonication, microwave irradiation, or the addition of suitable catalyst (Li et al., 2012).

\subsection{Dispersibility of NCC}

Generally, the dispersibility of NCC in a solvent system is mainly dependent on the surface charge of the particles and the ionic strength of the system (Camarero Espinosa et al., 2013; Dong, Revol, \& Gray, 1998). The dispersibility of NCC is of critical importance for its successful application. Fig. 4 shows the dispersibility of NCC and s-NCC dispersions in several different solvents (water, ethanol, and tetrahydrofuran) after sonication and standing for different times. The concentration of the prepared suspensions was $5 \mathrm{mg} / \mathrm{mL}$. As can be seen from Fig. 4, after sonication, both NCC and s-NCC could be well dispersed in water, ethanol, and tetrahydrofuran, respectively. However, there were clear aggregates of NCC in ethanol after $12 \mathrm{~h}$ standing, and obvious precipitation of NCC in both water and ethanol after $48 \mathrm{~h}$ standing, while s-NCC exhibited much better dispersibility in water and ethanol as presented in Fig. 4. This was due to the existing of a large amount of sulphate groups on the

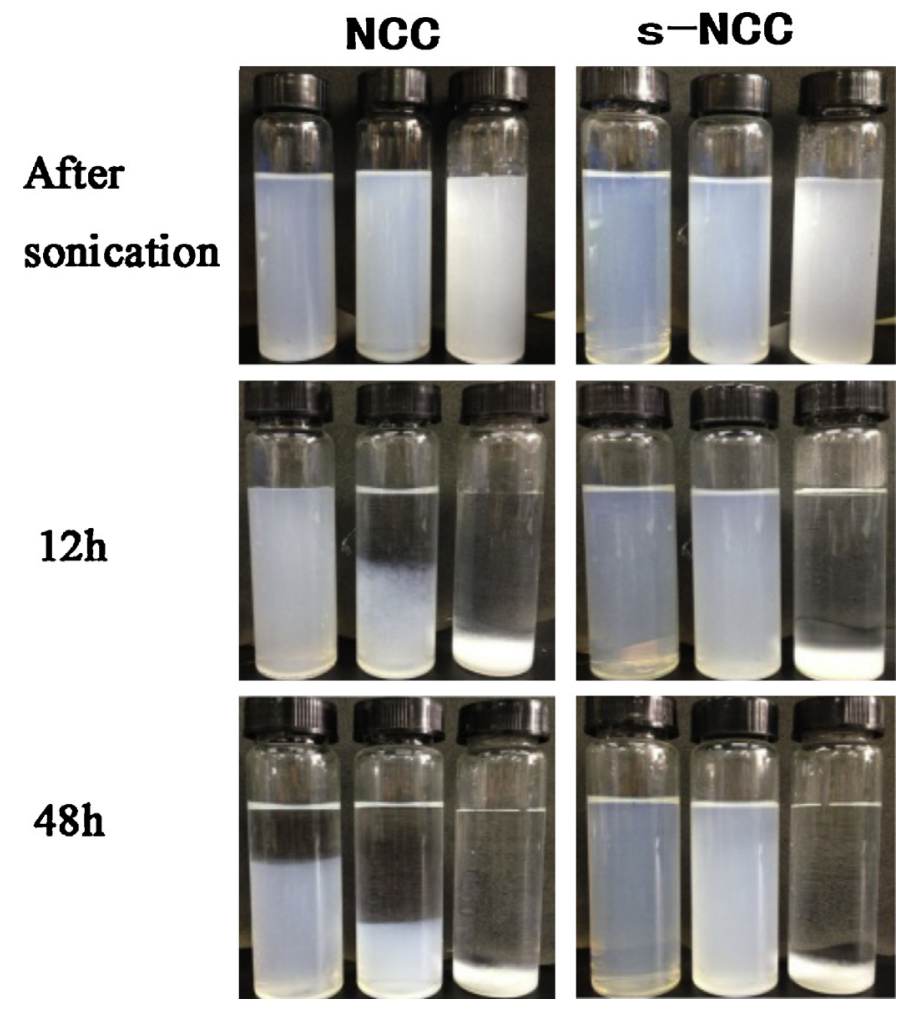

Fig. 4. Photographs of dispersions of NCC and s-NCC in water, ethanol and tetrahydrofuran (from left to right). In each case, the NCC concentration was $5 \mathrm{mg} / \mathrm{mL}$ Pictures were taken at $0 \mathrm{~h}, 12 \mathrm{~h}$, and $48 \mathrm{~h}$, respectively after sonication.

surface of s-NCC, thus generating a strong ionic repulsion between s-NCC particles (Habibi et al., 2010; Yu et al., 2013). Actually, the zeta potential of the prepared s-NCC and NCC suspension was about -20 and $-10 \mathrm{mV}$, respectively. Fig. 4 also shows that both NCC and s-NCC could easily aggregate in tetrahydrofuran due to the fact that their surface was highly hydrophilic. The good dispersibility of NCC in polar or non-polar solvents could be achieved by surface modification (Zaman, Xiao, Chibante, \& Ni, 2012; Zaman, Liu, Xiao, Chibante, \& Ni, 2013). In addition, it is worth pointing out that the aggregated NCC can be re-dispersed through ultrasonic treatment. A similar phenomenon was also found for the NCC prepared by hydrochloric acid hydrolysis under hydrothermal conditions (Yu et al., 2013).

\subsection{Morphology of NCC after evaporation-drying and freeze-drying}

Two different methods i.e. evaporation-drying and freezedrying were investigated to separate NCC from its suspension. FE-SEM shows that these two separation methods resulted in different morphology and microstructure of self-assembled NCC. After the complete evaporation of water from $2 \%$ NCC suspension, a transparent and flexible film was obtained (Fig. 5). While different types of NCC were obtained via ultrasonic-dispersion and freezedrying process. It was found that the suspension concentration significantly influenced the properties of the resultant cellulose nanofibers via ultrasonic processing, and the particle concentration in suspension was critical to the morphology of freeze-dried NCC, which is in agreement with previous reports (Chen et al., 2013; Deville, Saiz, Nalla, \& Tomsia, 2006). With concentration of $1.0 \%$, cellulose nanoparticles self-organized into fluffy foam composed of overlapping nanofiber layers, as shown in Fig. 6(a-c). In contrast, with concentration of $0.04 \%$, the NCC tended to self-assemble into ultrafine powder, which exhibited porous network structure at 

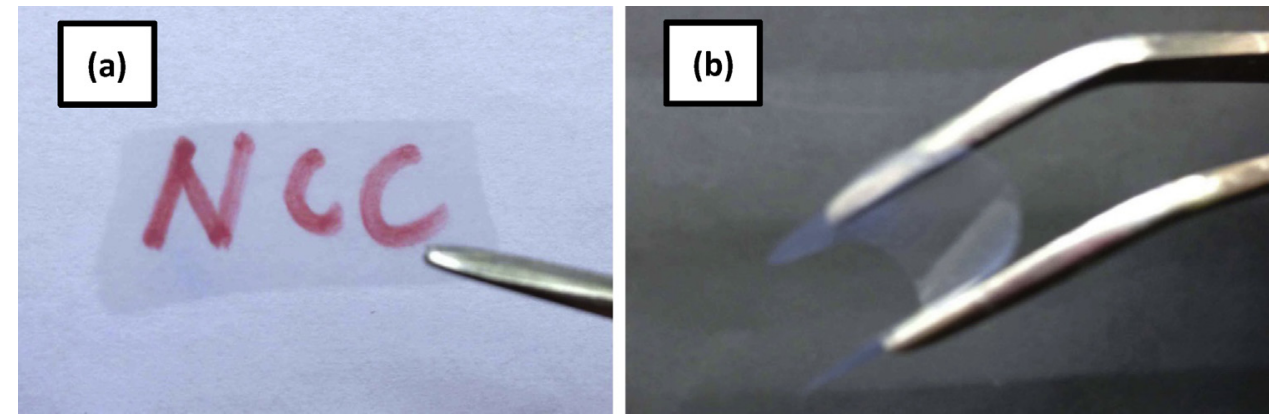

Fig. 5. Photographs of a transparent and flexible NCC film.

the micrometer scale, as shown in Fig. 6(d-f). These findings were consistent with the results reported by Han, Zhou, Wu, Liu, and Wu (2013).

\subsection{Chemical and crystalline structures of as-prepared NCC}

The X-ray diffractograms in Fig. 7a shows the diffraction patterns with diffraction peaks at around $2 \theta=14.9^{\circ}, 16.4^{\circ}, 22.7^{\circ}$ and $34.5^{\circ}$, corresponding to the (101), (1 10$),(200)$ and $(040)$ crystallographic planes of a typical cellulose I structure (Li \& Renneckar, 2011; Park, Baker, Himmel, Parilla, \& Johnson, 2010). Compared to the crystalline index of $65 \%$ for wood pulp sample, the crystallite index for NCC was $85 \%$. An increase of about $20 \%$ in crystallite should be attributed to the hydrolysis of amorphous regions in pulp. The average crystallite size of the pulp sample was $3.9 \mathrm{~nm}$ and that for the NCC was $5.8 \mathrm{~nm}$. The increased crystallite size of NCC mainly reflected the narrowing of the crystallite size distribution after hydrolysis (Lu \& Hsieh, 2012). Moreover, the $22.6^{\circ}$
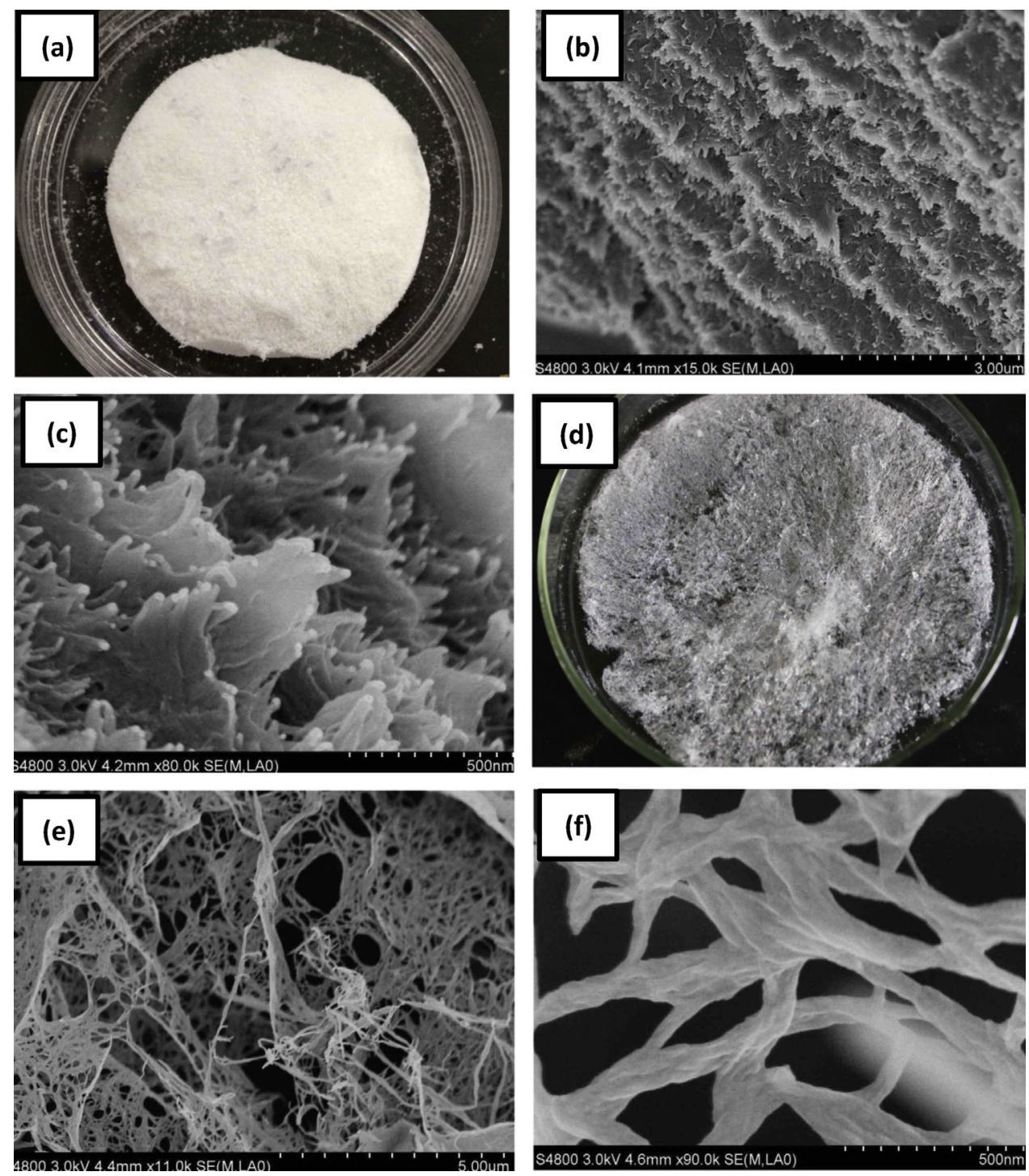

Fig. 6. Self-assembled NCCs obtained by lyophilization at different concentration. (a-c) $1.0 \%$ and (d-f) $0.04 \%$. 

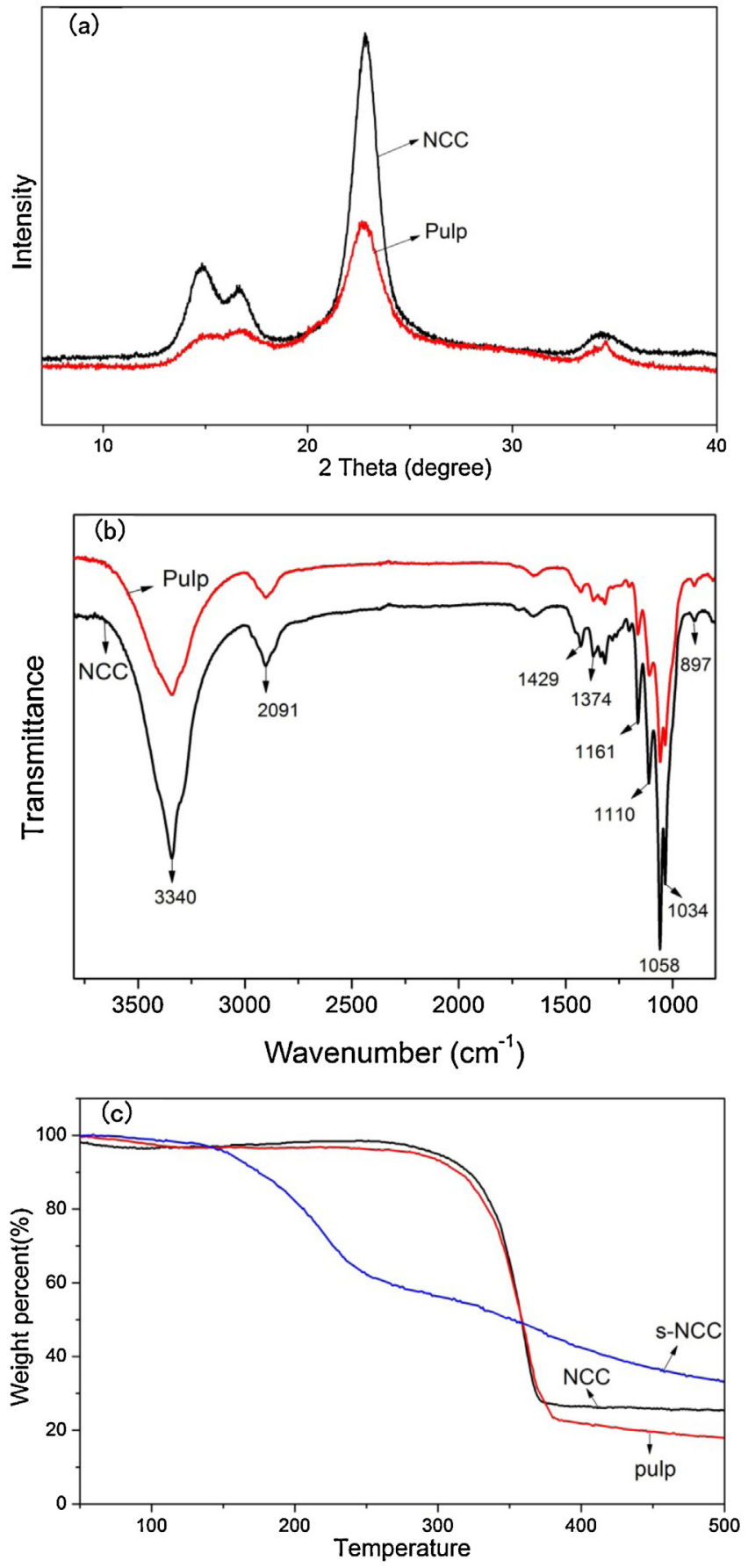

Fig. 7. (a) X-ray diffraction patterns of pulp and NCC, (b) FT-IR spectra of original wood pulp and NCC, (c) TG curves of pulp and NCCs obtained through different preparation routes.

corresponding to the (200) plane of NCC became shaper, indicating higher perfection of the crystal lattice in the $(200)$ plane than the original pulp. The peak at $2 \theta=15.0^{\circ}$ became more intense and was separated from the peak at $2 \theta=16.4^{\circ}$ for NCC, indicating a more compact and ordered crystalline structure.

FTIR spectra were used to characterize the chemical groups on the surface of NCC, as shown in Fig. 7b. The absorption bands at 3341 and $2901 \mathrm{~cm}^{-1}$ were assigned to $\mathrm{O}-\mathrm{H}$ stretching vibration and $\mathrm{C}-\mathrm{H}$ stretching vibration, respectively. The peaks at $1429,1161,1110$ and $897 \mathrm{~cm}^{-1}$ were thought to be the typical bonds of cellulose $I(I \beta)$ (Leung et al., 2011), which was consistent with XRD analysis. No
Table 2

The results of elemental analysis for pulp, fresh HPW and the regenerated HPW.

\begin{tabular}{lllll}
\hline Samples & $\mathrm{N} \%$ & $\mathrm{C} \%$ & $\mathrm{H} \%$ & $\mathrm{~S} \%$ \\
\hline Original pulp & 0.23 & 42.34 & 6.377 & 0.542 \\
Fresh HPW & 0.27 & 0.04 & 0.973 & 0.514 \\
HPW reused once & 0.22 & 0.04 & 0.762 & 0.252 \\
HPW reused twice & 0.44 & 0.03 & 1.236 & 0.539 \\
HPW reused three times & 0.36 & 0.08 & 1.090 & 0.485
\end{tabular}

significant difference was found in the spectrum of NCC compared to that of wood pulp.

\subsection{Thermal stability of NCCS}

Fig. 7c shows the TGA curves of the original bleached wood pulp, the NCC obtained from HPW hydrolysis, and the NCC obtained via traditional sulfuric acid hydrolysis, respectively. As can be seen, the NCC prepared by sulfuric acid hydrolysis had the lowest thermal stability and its decomposition temperature was about $245^{\circ} \mathrm{C}$. This was because the elimination of sulfuric acid in sulfated NCC required less energy (i.e. lower temperature) (Yu et al., 2013), and thus the released sulfuric acid would further accelerate the depolymerisation of cellulose by removing some of hydroxyl groups either by an esterification mechanism or by direct catalysis (Julien, Chornet, \& Overend, 1993; Roman \& Winter, 2004). In comparison, NCCs obtained from HPW hydrolysis exhibited much higher thermal stability, and its decomposition temperature was about $350^{\circ} \mathrm{C}$ (same as the wood pulp fibers). This was due to the lesser amount of damage in the crystalline regions and surface structure during the mild conditions of HPW hydrolysis process. The high thermal stability of NCC has large potential applications (Jonoobi, Harun, Mathew, \& Oksman, 2010; Oksman, Mathew, Bondeson, \& Kvien, 2006). For instance, the prepared NCC can be used as a filler in various materials or coatings that has high requirement of thermal stability (Hubbe, Rojas, Lucia, \& Sain, 2008).

\subsection{Reusability of HPW}

As illustrated in Fig. S2, the XRD pattern of the recovered HPW was consistent with the fresh HPW. According to the published research (Dias, Osegovic, \& Drago, 1999; Li et al., 2012), the crystal structure of HPW was primarily composed of the Keggin anion $\left(\left[\mathrm{PW}_{12} \mathrm{O}_{40}\right]^{3-}\right)$ (Keggin, 1933), and the Keggin structure of crystalline HPW was maintained in solution, turning out that the strength and behavior of the solvated HPW might be similar to those of solid HPW, which was due to the same Keggin anion in solution. The FTIR spectra (Fig. S3) clearly exhibited absorption bands in the $1100-850 \mathrm{~cm}^{-1}$ fingerprint region. The peaks at 1083 , 984,886 and $805 \mathrm{~cm}^{-1}$ were attributed to $v_{\text {as }}(\mathrm{P}-\mathrm{Oa}), v_{\mathrm{as}}(\mathrm{W}=\mathrm{Od})$, $v_{\text {as }}(\mathrm{W}-\mathrm{Ob}-\mathrm{W})$ and $\nu_{\mathrm{as}}(\mathrm{W}-\mathrm{Oc}-\mathrm{W})$ respectively, coinciding with the characteristic ones of the Keggin structure (Pizzio, Caceres, \& Blanco, 1998; Timofeeva et al., 2003). From the above analysis, it could be concluded that there were no significant changes in structure for the recovered HPW after each round of reaction. In addition, the elemental analyses tests for the fresh HPW and the regenerated HPW were performed. Four elementals (N, C, H, and S) were tested for each sample, and the measurement error from the instrument was about $0.1 \%$. According to Table 2 , the $C$ content of fresh HPW and the regenerated HPW could be considered as 0 , and thus we could conclude that there were no materials attached onto the regenerated HPW. The very little amount of $\mathrm{N}$ might be associated with the impurities in the carrier gas during tests, and the very small amount of $S$ might be associated with the impurities in the raw material. 

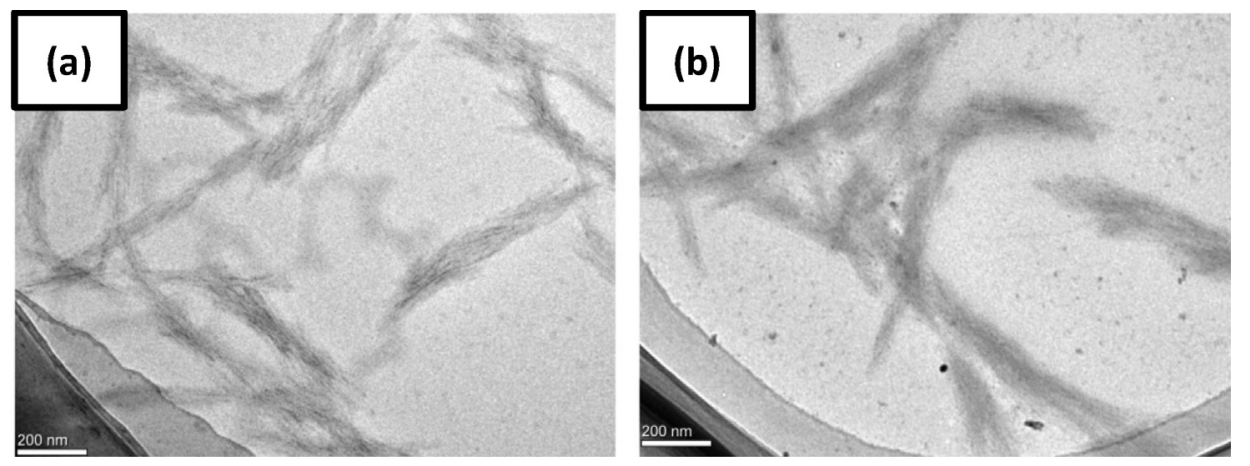

(c)

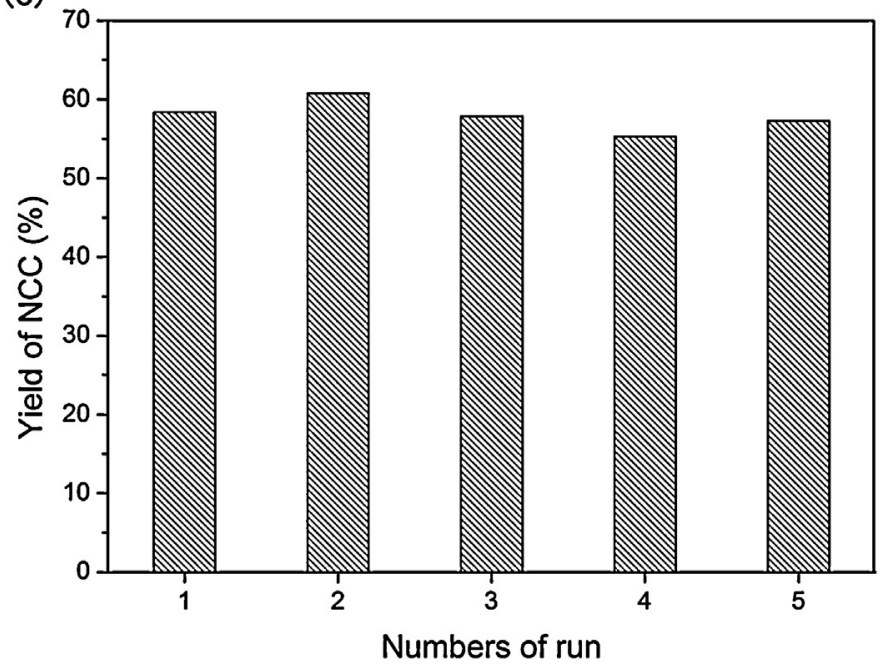

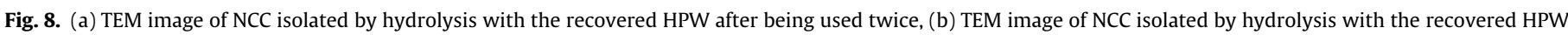
after being used five times, (c) the reusability of HPW for the hydrolysis of pulp under the same reaction conditions: $75 \% \mathrm{HPW}, 90{ }^{\circ} \mathrm{C}, 30 \mathrm{~h}$.

Therefore, the regenerated HPW should have similar reactivity to the fresh HPW. To verify this, the regenerated HPW was used to prepare NCC by the use of the same procedure as the fresh HPW. Based on the results obtained, the concentrated HPW as the catalyst could be recovered and reused for at least five times for cellulose hydrolysis to prepare isolated NCC. Morphology of NCC isolated by hydrolysis with the recovered HPW after several runs was similar to the ones isolated by the fresh HPW (Fig. 8a and b). The activity of the recycled HPW could be tested by real reaction. Fig. $8 \mathrm{c}$ shows that the catalytic activity of HPW in the 5 cycles' reuse remained almost unchanged. After reusing 5 times, the NCC yield still could reach about $58 \%$.

\section{Conclusions}

In this paper, a green and sustainable method for the preparation for nanocrystalline cellulose (NCC) from bleached hardwood pulp by the concentrated $\mathrm{H}_{3} \mathrm{PW}_{12} \mathrm{O}_{40}$ hydrolysis was established. It was found that the size of the obtained NCC particles after hydrolysis (75\% of $\mathrm{H}_{3} \mathrm{PW}_{12} \mathrm{O}_{40}$ at $90^{\circ} \mathrm{C}$ for $30 \mathrm{~h}$ ) was $15-40 \mathrm{~nm}$ in width and hundreds of nanometers in length. The prepared NCC had relatively good dispersibility in aqueous phase and high thermal stability. In addition, the used $\mathrm{H}_{3} \mathrm{PW}_{12} \mathrm{O}_{40}$ could be readily recovered and it exhibited consistently high performance in several runs of reaction.

\section{Acknowledgements}

We acknowledged the financial support by the National Natural Science Foundation of China (Nos. 21206814, 31370582, and
21306216), as well as the National High Technology Research and Development Program ("863" program) of China (No. 2012AA022301).

\section{Appendix A. Supplementary data}

Supplementary data associated with this article can be found, in the online version, at http://dx.doi.org/10.1016/j.carbpol. 2014.04.040.

\section{References}

Abraham, E., Deepa, B., Pothan, L. A., John, M., Narine, S. S., Thomas, S., et al. (2012). Physicomechanical properties of nanocomposites based on cellulose nanofibre and natural rubber latex. Cellulose, 20(1), 417-427.

Beck-Candanedo, S., Roman, M., \& Gray, D. G. (2005). Effect of reaction conditions on the properties and behavior of wood cellulose nanocrystal suspensions. Biomacromolecules, 6(2), 1048-1054.

Brown, E. E., Hu, D., Abu Lail, N., \& Zhang, X. (2013). Potential of nanocrystalline cellulose - fibrin nanocomposites for artificial vascular graft applications. Biomacromolecules, 14(4), 1063-1107.

Camarero Espinosa, S., Kuhnt, T., Foster, E. J., \& Weder, C. (2013). Isolation of thermally stable cellulose nanocrystals by phosphoric acid hydrolysis. Biomacromolecules, 14(4), 1223-1230.

Chen, P., Yu, H. P., Liu, Y. X., Chen, W. S., Wang, X. Q., \& Ouyang, M. (2013). Concentration effects on the isolation and dynamic rheological behavior of cellulose nanofibers via ultrasonic processing. Cellulose, 20(1), 149-157.

Deville, S., Saiz, E., Nalla, R. K., \& Tomsia, A. P. (2006). Freezing as a path to build complex composites. Science, 311(5760), 515-518.

Dias, J. A., Osegovic, J. P., \& Drago, R. S. (1999). The solid acidity of 12 tungstophosphoric acid. Journal of Catalysis, 183(1), 83-90.

Dong, X. M., Revol, J-F., \& Gray, D. G. (1998). Effect of microcrystallite preparation conditions on the formation of colloid crystals of cellulose. Cellulose, 5(1), 19-32.

Drago, R. S., Dias, J. A., \& Maier, T. O. (1997). An acidity scale for brönsted acids including $\mathrm{H}_{3} \mathrm{PW}_{12} \mathrm{O}_{40}$. Journal of the American Chemical Society, 119(33), 7702-7710. 
Eichhorn, S. J., Baillie, C. A., Zafeiropoulos, N., Mwaikambo, L. Y., Ansell, M. P., Dufresne, A., et al. (2001). Review: current international research into cellulosic fibres and composites. Journal of Materials Science, 36(9), 2107-2131.

Fan, J. S., \& Li, Y. H. (2012). Maximizing the yield of nanocrystalline cellulose from cotton pulp fiber. Carbohydrate Polymers, 88(4), 1184-1188.

Habibi, Y., Lucia, L. A., \& Rojas, O. J. (2010). Cellulose nanocrystals: Chemistry, selfassembly, and applications. Chemical Reviews, 110(6), 3479-3500.

Han, J. Q., Zhou, C. J., Wu, Y. Q., Liu, F. Y., \& Wu, Q. L. (2013). Self-assembling behavior of cellulose nanoparticles during freeze-drying: Effect of suspension concentration, particle size, crystal structure, and surface charge. Biomacromolecules, 14(5), 1529-1540.

Hebeish, A., Farag, S., Sharaf, S., \& Shaheen, T. I. (2013). Thermal responsive hydrogels Based on semi interpenetrating network of poly (NIPAm) and cellulose nanowhiskers. Carbohydrate Polymers, 102, 159-166.

Huang, J., Zhu, H. L., Chen, Y. C., Preston, C., Rohrbach, K., Cumings, J., et al. (2013). Highly transparent and flexible nanopaper transistors. ACS Nano, 7(3), 2106-2113.

Huang, Y. B., \& Fu, Y.(2013). Hydrolysis of cellulose to glucose by solid acid catalysts. Green Chemistry, 15(5), 1095-1111.

Hubbe, M. A., Rojas, O. J., Lucia, L. A., \& Sain, M. (2008). Cellulosic nanocomposites: A review. BioResources, 3(3), 929-980.

Jonoobi, M., Harun, J., Mathew, A. P., \& Oksman, K. (2010). Mechanical properties of cellulose nanofiber (CNF) reinforced polylactic acid (PLA) prepared by twin screw extrusion. Composites Science and Technology, 70(12), 1742-1747.

Julien, S., Chornet, E., \& Overend, R. P. (1993). Influence of acid pretreatment $\left(\mathrm{H}_{2} \mathrm{SO}_{4}\right.$ $\mathrm{HCl}, \mathrm{HNO}_{3}$ ) on reaction selectivity in the vacuum pyrolysis of cellulose. Journal of Analytical and Applied Pyrolysis, 27(1), 25-43.

Keggin, J. F. (1933). Structure of the molecule of 12-phosphotungstic acid. Nature, 131, 908-909.

Klemm, D., Kramer, F., Moritz, S., Lindstrom, T., Ankerfors, M., Gray, D., et al. (2011). Nanocelluloses: a new family of nature-based materials. Angewandte Chemie International Edition, 50(24), 5438-5466.

Kovacs, T., Naish, V., O'Connor, B., Blaise, C., Gagné, F., Hall, L., et al. (2010). An ecotoxicological characterization of nanocrystalline cellulose (NCC). Nanotoxicology, 4(3), 255-270.

Lavoine, N., Desloges, I., Dufresne, A., \& Bras, J. (2012). Microfibrillated cellulose Its barrier properties and applications in cellulosic materials: A review. Carbohydrate polymers, 90(2), 735-764.

Leung, A. C., Hrapovic, S., Lam, E., Liu, Y. L., Male, K. B., Mahmoud, K. A., et al. (2011). Characteristics and properties of carboxylated cellulose nanocrystals prepared from a novel one-step procedure. Small, 7(3), 302-305

Li, X., Jang, Y., Wang, L., Meng, L., Wang, W., \& Mu, X. (2012). Effective lowtemperature hydrolysis of cellulose catalyzed by concentrated $\mathrm{H}_{3} \mathrm{PW}_{12} \mathrm{O}_{40}$ under microwave irradiation. RSC Advances, 2, 6921-6925.

Li, Q., \& Renneckar, S. (2011). Supramolecular structure characterization of molecularly thin cellulose I nanoparticles. Biomacromolecules, 12(3), 650-659.

Lu, P., \& Hsieh, Y. L. (2012). Preparation and characterization of cellulose nanocrystals from rice straw. Carbohydrate Polymers, 87, 564-573.

Oksman, K., Mathew, A. P., Bondeson, D., \& Kvien, I. (2006). Manufacturing process of cellulose whiskers/polylactic acid nanocomposites. Composites Science and Technology, 66(15), 2776-2784.

Park, S., Baker, J. O., Himmel, M. E., Parilla, P. A., \& Johnson, D. K. (2010). Cellulose crystallinity index: Measurement techniques and their impact on interpreting cellulose performance. Biotechnology for Biofuels, 3, 10 http://dx.doi.org/10.1186/1754-6834-3-10

Pizzio, L. R., Caceres, C. V., \& Blanco, M. N. (1998). Acid catalysts prepared by impregnation of tungstophosphoric acid solutions on different supports. Applied Catalysis A: General, 167(2), 283-294.

Roman, M., \& Winter, W. T. (2004). Effect of sulphate groups from sulphuric acid hydrolysis on the thermal degradation behaviour of bacterial cellulose. Biomacromolecules, 5(5), 1671-1677.

Segal, L., Creely, J., Martin, A., \& Conrad, C. (1959). An empirical method for estimating the degree of crystallinity of native cellulose using the X-ray diffractometer. Textile Research Journal, 29(10), 786-794.

Shen, J., Song, Z., Qian, X., \& Ni, Y. (2011). Carbohydrate-based fillers and pigments for papermaking: A review. Carbohydrate Polymers, 85(1), 11-22.

Shimizu, K., Furukawa, H., Kobayashi, N., Itaya, Y., \& Satsuma, A. (2009). Effects of Brønsted and Lewis acidities on activity and selectivity of heteropolyacid-based catalysts for hydrolysis of cellobiose and cellulose. Green Chemistry, 11(10), 1627-1632.

Shopsowitz, K. E., Hamad, W. Y., \& MacLachlan, M. J. (2011). Chiral nematic mesoporous carbon derived from nanocrystalline cellulose. Angewandte Chemie International Edition, 50(46), 10991-10995.

Tang, L., Huang, B., Ou, W., Chen, X., \& Chen, Y. (2011). Manufacture of cellulose nanocrystals by cation exchange resin-catalyzed hydrolysis of cellulose. Bioresource Technology, 102, 10973-10977.

Tang, Y., Yang, S., Zhang, N., \& Zhang, J. (2014). Preparation and characterization of nanocrystalline cellulose via low-intensity ultrasonic-assisted sulfuric acid hydrolysis. Cellulose, 21, 335-346.

Teixeira, E. M., de Oliveira, C. R., Mattoso, L. H. C., Corrêa, A. C., \& Paladin, P. D. (2010). Nanofibras de algodão obtidas sob diferentes condições de hidrólise ácida. Polímeros, 20(4), 264-268.

Timofeeva, M. N., Matrosova, M. M., Il'inich, G. N., Reshetenko, T. V., Avdeeva, L. B., Kvon, R. I., et al. (2003). Esterification of $n$-butanol with acetic acid in the presence of $\mathrm{H}_{3} \mathrm{PW}_{12} \mathrm{O}_{40}$ supported on mesoporous carbon materials. Kinetics and Catalysis, 44(6), 778-787.

Trolliet, C., Coudurier, G., \& Védrine, J. C. (2001). Influence of the nature and porosity of different supports on the acidic and catalytic properties of $\mathrm{H}_{3} \mathrm{PW}_{12} \mathrm{O}_{40}$. Topics in Catalysis, 15(1), 73-81.

Wang, Q. Q., Zhu, J. Y., Reiner, R. S., Verrill, S. P., Baxa, U., \& McNeil, S. E. (2012) Approaching zero cellulose loss in cellulose nanocrystal (CNC) production: Recovery and characterization of cellulosic solid residues (CSR) and CNC. Cellulose, 19(6), 2033-2047.

Yousefi, H., Nishino, T., Faezipour, M., Ebrahimi, G., \& Shakeri, A. (2011). Direct fabrication of all-cellulose nanocomposite from cellulose microfibers using ionic liquid-based nanowelding. Biomacromolecules, 12(11), 4080-4085.

Yu, H. Y., Qin, Z. Y., Liang, B. L., Liu, N., Zhou, Z., \& Chen, L. (2013). Facile extraction of thermally stable cellulose nanocrystals with a high yield of $93 \%$ through hydrochloric acid hydrolysis under hydrothermal conditions. Journal of Materials Chemistry A, 1, 3938-3944.

Zaman, M., Liu, H., Xiao, H., Chibante, F., \& Ni, Y. (2013). Hydrophilic modification of polyester fabric by applying nanocrystalline cellulose containing surface finish. Carbohydrate Polymers, 91, 560-567.

Zaman, M., Xiao, H., Chibante, F., \& Ni, Y. (2012). Synthesis and characterization of cationically modified nanocrystalline cellulose. Carbohydrate Polymers, 89 163-170. 